\title{
Signal Processing for Phased Array Feeds in Radio Astronomical Telescopes
}

\author{
Brian D. Jeffs, Senior Member, IEEE, Karl F. Warnick Senior Member, IEEE, \\ Jonathan Landon, Jacob Waldron, David Jones, J. Richard Fisher $\dagger$, Roger D. Norrod $\ddagger$
}

\begin{abstract}
Relative to traditional waveguide feeds, phased array feeds (PAFs) for radio telescopes can increase the instrument field of view and sky survey speed. Unique challenges associated with PAF observations, including extremely low signal levels, long-term system gain stability requirements, spatially correlated noise due to mutual coupling, and tight beamshape tolerances, require the development of new array signal processing techniques for this application. We propose a calibration and beamforming strategy for PAFs including interference mitigation with power spectral density (PSD) estimation bias correction. Key efficiency metrics for single-feed instruments are extended to the array case and used to verify performance of the algorithms. These techniques are validated using numerical simulations and experimental data from a 19 element PAF on the Green Bank 20-Meter Telescope.
\end{abstract}

Index Terms-Adaptive arrays, Interference suppression, Radio Astronomy

\section{INTRODUCTION}

From the earliest days of radio astronomy (RA), detecting faint deep space sources has pushed available technology to extreme performance limits. Early progress was driven by improvements in hardware [1], [2] with relatively straightforward signal processing and detection techniques. With the advent of large synthesis arrays, signal processing algorithms increased in sophistication [3]. More recently, interest in phased array feeds (PAFs) has opened a new frontier for array signal processing algorithm development for RA observations.

Array feeds in use at present consist of multiple traditional waveguide feed horn antennas providing independent sky mapping pixels with low main beam overlap [4]. The feeds are typically not processed jointly as a sensor array. Since waveguide feeds are physically large, the elements cannot be packed tightly enough to provide a continuous image in one snapshot. To achieve continuous sky coverage and greater control over beam patterns, several research groups are developing phased array feeds (PAFs) consisting of closely spaced, electrically small elements [5]-[8]. Such an array provides a number of advantages over traditional feeds, including multiple steered beams, sensitivity optimization with respect to the noise environment, and interference cancelation.

Phased array feeds are already in use for communications applications, but for RA, PAF development has been

This work was funded by National Science Foundation under grant number AST - 0352705

The authors are with the Department of Electrical and Computer Engineering, Brigham Young University, Provo, UT 84602 USA (bjeffs@ee.byu.edu, warnick@ee.byu.edu), except $\dagger$ : National Radio Astronomy Observatory (NRAO), Charlottesville, VA 22903-2475, rfisher@nrao.edu, ł: NRAO, Green Bank, WV 24944, rnorrod@nrao.edu slower. NRAO developed an early 19 element array of sinuous antennas [5]. The Netherlands Foundation for Research in Astronomy (ASTRON) has demonstrated good illumination efficiency for a wideband, dual polarized Vivaldi array [8]. The Canadian National Research Council has developed a Phased Array Feed Demonstrator (PHAD), also of Vivaldi elements [7]. The Australian Commonwealth Scientific and Industrial Research Organization (CSIRO) is developing a wideband connected checkerboard array. The Karoo Array Telescope (meerKAT) being developed in South Africa will be upgraded to a PAF implementation. ASKAP and meerKAT are Square Kilometer Array (SKA) pathfinder instruments [9].

Brigham Young University and NRAO have had a collaborative effort in PAF development since 2003. We first successfully demonstrated beamforming and RFI mitigation with a seven element hexagonal array on a three meter reflector in 2006 [10]. A 19 element, L-band dipole array was deployed on the NRAO Green Bank 20-Meter Telescope in 2007 for a series of experiments to measure aperture and spillover efficiency, demonstrate multiple beam formation, and test RFI mitigation algorithms with real and simulated interference sources (see Figure 1). Experimental results from the 19 element array are reported in this paper.

Due to extremely low signal levels and high stability requirements for astronomical instruments, successful application of PAFs in radio astronomy requires the development of new array signal processing strategies. In this paper, we develop a calibration procedure and a "fixed-adaptive" beamforming approach for PAFs that delivers sufficient gain and sensitivity for radio observations while maintaining control over beam pattern sidelobes and system noise. To verify performance of the algorithms, we extend efficiency metrics used for single-feed instruments to the array case. Radio frequency interference (RFI) is a significant and growing problem for astronomical observations, but adaptive interference cancelation is not compatible with the high gain stability requirements of radio astronomy due to pattern rumble. We apply a power spectral density (PSD) pattern rumble bias correction algorithm to a 19 element focal plane array and verify the performance of the algorithm using numerical simulations.

In addition to presenting these new results on array signal processing for PAFs, a second purpose of this paper is to define the radio astronomy phased array feed signal processing problem as an important regime for algorithm development work, particularly in view of the planned future synthesis imaging arrays of medium sized dishes that will use PAFs. The emphasis is on unique challenges as compared to typical 


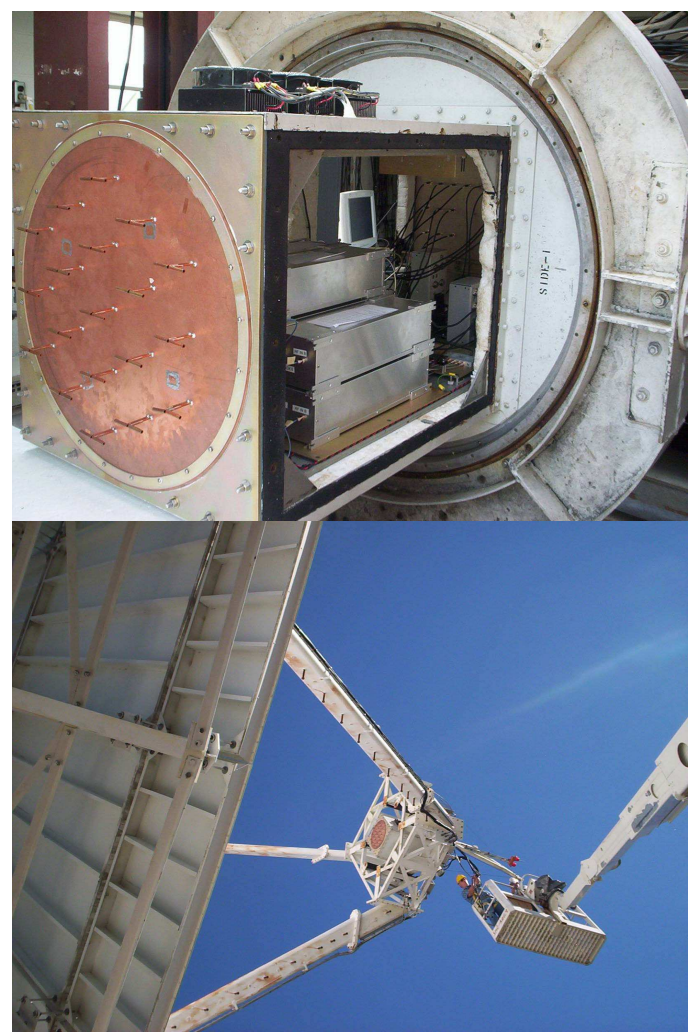

Fig. 1. Top: 19 element BYU/NRAO L-band PAF and front end box with low noise amplifiers and downconverters behind the array. Bottom: PAF mounted on Green Bank 20-Meter Telescope (October, 2007).

applications in communications, radar, sonar, or remote sensing. These issues, and the models, analysis, and experimental results presented here, apply both to single dish telescopes and interferometric arrays of many dishes, each with a PAF at its focal plane. Some of the special conditions encountered in astronomical signal processing are:

1) Radiometric detection: A basic observational mode in RA is "on-source minus off-source" radiometric detection. This requires stable power estimates of $i$ ) system noise plus weak signal of interest, and ii) noise power alone with the sensor steered off the signal of interest. The standard deviation of the noise power estimate determines the minimum detectable signal level, so that long integration times (minutes to hours) are required.

2) Low SNR: Deep space signals are extremely faint. SNRs of -30 to $-50 \mathrm{~dB}$ are routine.

3) Low system temperatures: With cryo-cooled LNAs, receiver noise temperatures can be as low as $5 \mathrm{~K}$ at Lband, including LNA noise, waveguide ohmic losses, and downstream receiver noise. With high spillover efficiency and low PAF pattern sidelobes, sky and spillover noise increase system temperatures nominally to $15 \mathrm{~K}$. Traditionally, this is an antenna design issue, but for PAFs it becomes an array signal processing problem.

4) Stability: System gain fluctuations increase the receiver output variance and place a limit on achievable sensitivity that cannot be overcome with increased integration time. For a PAF, response fluctuations caused by beam- former processing must be controlled or mitigated.

5) Calibration: Sensitive observations will require wellcalibrated beamformers. Optimal performance will be achieved with periodic calibration on measured responses for bright astronomical sources to compensate for receiver phase and amplitude drifts.

6) Bandwidth: Some scientific observations require broad bandwidths of an octave or more. Digital beamforming over such large bandwidths poses a serious challenge.

7) Mutual coupling: Strong electrical coupling between closely packed array elements leads to increased noise correlation and affects the optimal beamformer for a given observation scenario.

8) Radio frequency interference (RFI): Observations in RFI environments outside protected frequency bands are common. Interference levels below the noise floor may be as problematic as strong interferers, since they are hard to identify and attenuate. Cancellation approaches also cause pattern rumble which limits sensitivity.

The goals of this paper are to present new results addressing these signal processing challenges for PAFs, and to motivate further progress in hardware and algorithm development needed to realize science-ready array feeds. Section II presents mathematical models for signal and noise, and describes our calibration procedure. Section III discusses challenges of weak signal detection in the RA environment and derives expressions for antenna performance metrics such as sensitivity and efficiencies. Use of PAFs for mitigating interference is addressed in Section V. Concluding remarks are found in Section VI, and notation is defined in the Appendix.

\section{SignAl MODEL}

\section{A. Sensor Array and Beamforming}

We assume narrowband operation so $B \ll D / c$, where $B$ is bandwidth, $D$ is the PAF diameter, and $c$ is the speed of light. As illustrated in Figure 2, the $P$ element PAF produces a length $P \times 1$ baseband data vector at time sample $n$ :

$$
\mathbf{x}[n]=\mathbf{a} s[n]+\sum_{q=1}^{Q} \mathbf{v}_{q}[n] d_{q}[n]+\mathbf{n}[n]
$$

where $s[n]$ is the signal of interest (SOI), $\mathbf{n}[n]$ is noise, and $d_{q}[n]$ is one of $Q$ "detrimental" interfering sources. Vectors $\mathbf{a}$ and $\mathbf{v}_{q}[n]$ are normalized array responses to unit amplitude point sources in the far field at directions corresponding to $s[n]$ and $d_{q}[n]$ respectively. $\mathbf{v}_{q}[n]$ is non-stationary over the long term due to interferer motion. Since motion is relatively slow compared to the sample rate, over $L$ time samples called the "short term integration (STI)" window, the $\mathbf{v}_{q}[n]$ are approximately constant. Large bandwidths of interest in RA (e.g. up to an octave) can be handled by subband processing, computing successive windowed FFTs for each sensor, and repeating the architecture of Figure 2 in each frequency bin.

Assuming zero mean signals, the sample covariance matrix 


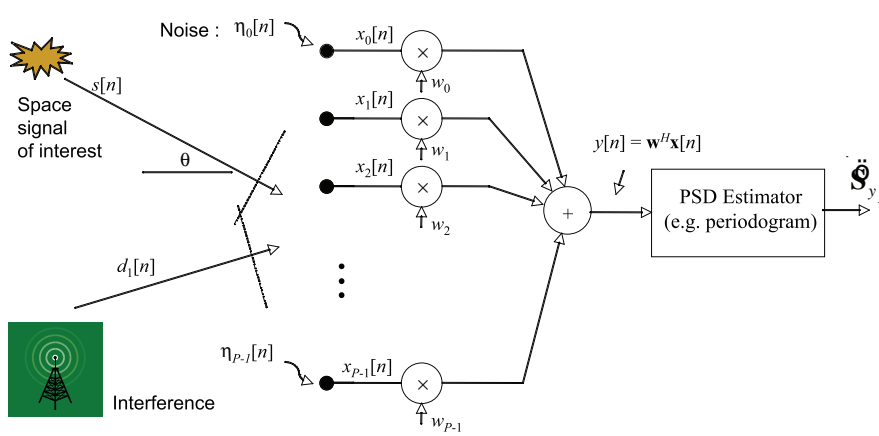

Fig. 2. Block diagram for signal processing of an example PAF application, including an adaptive beamformer to cancel interference, followed by power spectral density (PSD) estimation.

for the $j$ th STI window is defined as

$$
\begin{aligned}
\hat{\mathbf{R}}_{x, j} & =\frac{1}{L} \sum_{n=j L}^{(j+1) L-1} \mathbf{x}[n] \mathbf{x}^{H}[n]=\frac{1}{L} \mathbf{X}_{j} \mathbf{X}_{j}^{H} \\
\mathbf{X}_{j} & =[\mathbf{x}[j L], \mathbf{x}[j L+1], \cdots, \mathbf{x}[(j+1) L-1]] .
\end{aligned}
$$

The multiple beamformer output is formed by

$$
y_{i}[n]=\mathbf{w}_{i, j}^{H} \mathbf{x}[n], \quad 0 \leq i \leq K, \quad j=\lfloor n / L\rfloor .
$$

where a distinct $\mathbf{w}_{i, j}$ is computed for each mainlobe steering angle, $\Omega_{i}$. For fixed beamforming, $\mathbf{w}_{i, j}$ will not depend on $j$. For adaptive interference canceling or sensitivity optimization, $\mathbf{w}_{i, j}$ is recomputed at each STI window based on covariance estimate $\hat{\mathbf{R}}_{x, j}$ using any one of the beamformer methods described in section $\mathrm{V}$.

In a practical PAF scenario the beams are steered in a hexagonal grid pattern with crossover points at the -1 to -3 $\mathrm{dB}$ levels. The total number of beams, $K$, is limited by the maximum steering angle (which is determined by the diameter of the array feed), by the acceptable limit for beam distortion, or coma, and by the available processing capacity.

\section{B. Noise Model}

Because of the importance of low system noise for astronomical instruments, a sufficiently detailed model for $\mathbf{n}[n]$ is essential to signal processing algorithm studies. For phased array feeds, the correlation structure of the noise is particularly important, and a simple, uncorrelated noise model is inadequate. Noise components for a typical reflector antenna system are $i$ ) spillover noise from the warm ground seen by the feed beyond the rim of the obscuring reflector dish, ii) sky noise from the atmosphere and cosmic background radiation, iii) noise caused by ohmic losses in antenna elements, and iv) receiver noise due mainly to front end amplifiers. Since the spillover noise arrives at the feed from a limited angular region, it is strongly correlated across the array. Front end amplifier noise is also strongly correlated due to mutual coupling between array elements. The beamformer must account for this correlation to achieve an optimal reflector illumination pattern and reduce spillover and receiver noise. In general, the noise correlation matrix has the form

$$
\mathbf{R}_{\mathbf{n}}=\mathrm{E}\left\{\mathbf{n} \mathbf{n}^{H}\right\}=\mathbf{R}_{\mathrm{sp}}+\mathbf{R}_{\mathrm{sky}}+\mathbf{R}_{\mathrm{loss}}+\mathbf{R}_{\mathrm{rec}}
$$

where $\mathbf{R}_{\mathrm{sp}}$ is the spillover noise correlation matrix, $\mathbf{R}_{\text {sky }}$ is the sky noise contribution, $\mathbf{R}_{\text {loss }}$ represents thermal noise from the antenna elements, and $\mathbf{R}_{\text {rec }}$ is due to noise added by the front end amplifiers and receiver chains.

A numerical model for an array feed system can be developed using antenna analysis techniques and microwave network theory. By the electromagnetic reciprocity principle, the signal response $\mathbf{a}_{\Omega}$ of the array can be obtained from the embedded array element radiation patterns $\bar{E}_{m}(\Omega)$, which are defined by driving the $m$ th element with a unit input current excitation while the other element terminals are open circuited. The spillover noise response can be obtained by integrating the element patterns with respect to the angular thermal noise distribution. The spillover noise correlation matrix is [11]

$$
\mathbf{R}_{\mathrm{sp}}=16 k_{b} T_{\text {ground }} B \mathbf{Q} \mathbf{A}_{\mathrm{sp}} \mathbf{Q}^{H}
$$

where $k_{b}$ is Boltzmann's constant and $B$ is the system noise equivalent bandwidth (noise temperature in degrees Kelvin is related to the noise power spectral density by $k_{b} T$ ). $\mathbf{Q}$ is the relationship between open circuit voltages at the antenna terminals to receiver output voltages and can be obtained using network theory [12]. $\mathbf{A}_{\mathrm{sp}}$ is a matrix of pattern spillover overlap integrals given by

$$
A_{\mathrm{sp}, m n}=\int_{\Omega_{s p}} \bar{E}_{m} \cdot \bar{E}_{n}^{*} d \Omega
$$

where $\Omega_{\mathrm{sp}}$ is the solid angle from the reflector rim to the horizon. The receiver noise $\mathbf{R}_{\text {rec }}$ can be modeled using the network approach of [12], [13]. Array mutual coupling leads to correlation of front end amplifier noise and $\mathbf{R}_{\mathrm{rec}}$ is in general non-diagonal. Sky noise $\mathbf{R}_{\text {sky }}$ is usually less important than other contributions at microwave frequencies and will be neglected here. In the numerical results presented in this paper, the elements will be modeled as lossless, so that $\mathbf{R}_{\text {loss }}=0$.

\section{Calibration}

Due to strict beampattern stability requirements, it will be necessary to perform periodic calibration on the array to correct for electronic phase and gain responses which may drift differentially over time. Characterization of changes in the LNA noise temperatures is also important.

Bench-top pre-calibration is of no practical use beyond gross gain characterization. Calibration must include all reflector, element pattern, mutual coupling, and array support structure effects. The reference source must appear in the far field with no multipath, so for the large instruments in question, it is not realistic to provide a fixed man-made source. Long integrations on the brightest available deep-space sources as calibrators are required. In the northern hemisphere the two brightest continuum (broadband) calibrator sources are supernova remnant Cassiopeia A and radio galaxy Cygnus A.

Since multiple simultaneous beams are possible with a PAF, calibration must be performed for each direction, $\Omega_{i}$, corresponding to a beam's boresight, and any additional directions where point constraints in the beampattern response will be placed. Our proposed calibration algorithm is as follows:

1) Steer the dish to a relatively empty patch of sky so $\mathbf{x}[n]=\mathbf{n}[n]$, and collect a long term (large $L$, e.g. 10 
minutes) sample covariance estimate for the noise field, $\hat{\mathbf{R}}_{\mathbf{n}}$ using (1).

2) While tracking the brightest available calibration point source, steer the dish to calibration angle $\Omega_{i}$ (relative to this source). The observed signal model is $\mathbf{x}[n]=$ $\mathbf{a}_{\Omega_{i}} s[n]+\mathbf{n}[n]$, where $s[n]$ is the calibrator source signal and $\mathbf{a}_{\Omega_{i}}$ is the desired calibration vector. Calculate $\hat{\mathbf{R}}_{\Omega_{i}}$ using (1) and the same $L$ as in step 1 .

3) Repeat step 2 in a grid pattern corresponding to the desired distribution of beam centers and constraint points, e.g. for $\left\{\Omega_{i} \mid 1 \leq i \leq K\right\}$.

4) The estimated calibration vector is given by $\hat{\mathbf{a}}_{\Omega_{i}}=\mathbf{u}_{1}$, where $\mathbf{u}_{1}$ is the dominant eigenvector of $\left(\hat{\mathbf{R}}_{\Omega_{i}}-\hat{\mathbf{R}}_{\mathbf{n}}\right)$.

It is necessary in step three to subtract off $\hat{\mathbf{R}}_{\mathbf{n}}$ since noise is strongly correlated and even with long integration times, the available calibrator sources do not dominate the noise subspace sufficiently to keep $\mathbf{n}[n]$ from perturbing $\hat{\mathbf{a}}_{\Omega_{i}}$.

\section{Signal DETECTION}

Detecting a signal that is many $\mathrm{dB}$ below the noise floor requires accurate power estimates of the noise plus signal power (an "on" measurement) and the noise alone ("off" measurement). Differencing of the two measurements yields a signal power estimate. The minimum detectable signal level is determined by the standard deviation $\Delta T$ of the noise temperature (power) estimate. If the system gain is stable, it is well known that $\Delta T$ decreases inversely as the square root of the integration time. If the receiver gain is not stable and gain variability is not compensated for by calibration, then $\Delta T$ decreases initially but asymptotically approaches a fixed limit according to [1], [2]

$$
\Delta T=T_{\text {sys }} \sqrt{\frac{1}{B t}+\left(\frac{\Delta G}{\bar{G}}\right)^{2}}
$$

where $\Delta G / \bar{G}$ is the standard deviation of the gain relative to the mean. $T_{\text {sys }}$ is the system noise temperature in Kelvin, which by convention is referred to available power at the antenna terminals.

To illustrate the integration and stability requirements for astronomical observations, a moderately intense radio source may have an intensity of $50 \mathrm{mJy}\left(1 \mathrm{Jy}=10^{-29} \mathrm{~W} / \mathrm{m}^{2} / \mathrm{Hz}\right)$. The gain of a 20 meter reflector antenna is approximately $70 \mathrm{mK} / \mathrm{Jy}$, so the equivalent antenna temperature due to the signal is $3.5 \mathrm{mK}$. A typical system noise temperature at Lband using cryo-cooled LNAs is $20 \mathrm{~K}$, which implies an SNR of $-38 \mathrm{~dB}$. At a processing bandwidth of $B=10 \mathrm{KHz}$, detecting the signal requires roughly one hour of integration time. Solving (6) for $\Delta G / \bar{G}$ indicates system gain, including variation or pattern rumble due to adaptive beamforming, must be stable to better than one part in $6 \times 10^{3}$. Clearly, weak sources pose a significant detection challenge for a PAF, since the beamformer response must be stable enough to allow very long integration times.

An example of a spectral line observation is shown in Figure 3, using data from the BYU/NRAO 19 element array feed on the Green Bank 20-Meter Telescope. Sky catalogue object $\mathrm{W} 49 \mathrm{~N}$ is a strong $\mathrm{OH}$ maser source and can be detected with a relatively short integration time. Even so, in the onsource power spectral density (PSD) the signal is a small perturbation of the noise floor. Only by subtracting the on and off measurements can the spectral peak be identified near 1665.27 MHz.

This power-differencing mode of detection has implications in the array signal processing approaches which may be applied. Noise floor response stability between on-source and off-source beamforming is critical. Typical minimum variance methods (e.g. LCMV) may introduce variations in noise responses while minimizing total power in the presence of a variable or moving interferer. Pattern rumble correction may be required, as discussed in Section V-C.

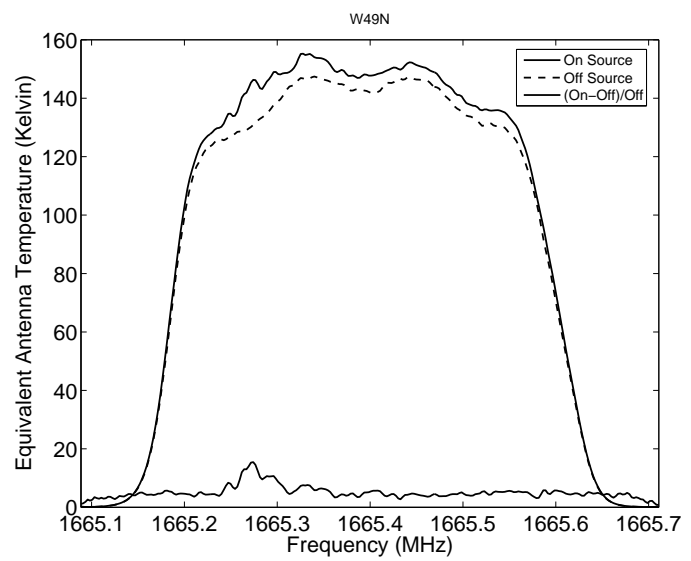

Fig. 3. On source, noise only, and relative difference power spectral densities for astronomical $\mathrm{OH}$ maser source $\mathrm{W} 49 \mathrm{~N}$ observed using the BYU/NRAO 19 element array feed on the Green Bank 20-Meter Telescope. The source intensity at the peak near $1665.28 \mathrm{MHz}$ is approximately $200 \mathrm{Jy}$.

\section{BEAM SENSITIVITY AND EFFICIENCIES}

The key performance metric for an array feed is SNR at the beamformer output, which is [11], [14]

$$
\mathrm{SNR}=\frac{\mathbf{w}^{H} \mathbf{R}_{\mathrm{s}} \mathbf{w}}{\mathbf{w}^{H} \mathbf{R}_{\mathbf{n}} \mathbf{w}}
$$

where $\mathbf{R}_{\mathrm{s}}=\sigma_{s}^{2} \mathbf{a a}^{H}$ is the signal of interest correlation matrix. The dependence of the SNR on the signal flux density is commonly removed to obtain "sensitivity," defined as the effective receiving area relative to the system noise temperature,

$$
\frac{A_{\mathrm{e}}}{T_{\mathrm{sys}}}=\frac{k_{b} B}{F_{\mathrm{s}}} \frac{\mathbf{w}^{H} \mathbf{R}_{\mathrm{s}} \mathbf{w}}{\mathbf{w}^{H} \mathbf{R}_{\mathbf{n}} \mathbf{w}} \quad\left(\mathrm{m}^{2} / \mathrm{K}\right)
$$

where $F_{\mathrm{S}}\left(\mathrm{W} / \mathrm{m}^{2}\right)$ is the signal flux density in one polarization. For a reflector antenna with a traditional horn feed, maximizing sensitivity involves a hardware-only tradeoff between aperture efficiency, which determines the received signal power, and spillover efficiency, which determines the spillover noise contribution. With a PAF, sensitivity is determined by the beamforming algorithm as well as the array and receivers.

To facilitate the joint hardware and algorithm design process required to optimize a PAF system, it is desirable to extend the figures of merit used in single-feed antenna design work, including aperture efficiency, radiation efficiency, and spillover 
efficiency, to an array sensor. Using the electromagnetic reciprocity principle, there is a direct relationship between the power radiated by a transmitting antenna and the noise power received by the same antenna in a spatially isotropic thermal noise field, which allows these efficiency definitions to be extended in a rigorous way to receiving arrays [11], [15].

For a lossless, passive antenna, aperture efficiency is the signal power received relative to the signal power incident on the antenna. Since the signal output power for an array is scaled by receiver gains and beamformer weights, the beamformer output must be normalized to remove this scaling before computing the aperture efficiency. For a passive antenna in an isotropic noise field with brightness temperature $T_{\text {iso }}$, the available noise power at the antenna terminals is $k_{b} T_{\text {iso }} B$. By scaling the beamformer output to have the same property, aperture efficiency can be defined for an array as

$$
\eta_{\text {ap }}=\frac{P_{\mathrm{s}}}{P_{\text {inc }}}=\frac{k_{b} T_{\text {iso }} B}{A_{\text {ap }} F_{\mathrm{s}}} \frac{\mathbf{w}^{H} \mathbf{R}_{\mathrm{s}} \mathbf{w}}{\mathbf{w}^{H} \mathbf{R}_{\text {iso }} \mathbf{w}}
$$

where $A_{\text {ap }}$ is the physical area of the aperture and $P_{\text {inc }}=$ $A_{\mathrm{ap}} F_{\mathrm{s}}$. The response of the array to an isotropic thermal noise field at temperature $T_{\text {iso }}$ up to a scale factor is

$$
\mathbf{R}_{\text {iso }}=16 k_{b} T_{\text {iso }} B \mathbf{Q A} \mathbf{A Q}^{H}
$$

where the pattern overlap integral matrix $\mathbf{A}$ has elements given by (5) but with the integral evaluated over a full sphere.

Using (10) and (4), the beam spillover efficiency can be expressed as

$$
\eta_{\mathrm{sp}}=1-\frac{T_{\text {iso }}}{T_{\mathrm{sp}}} \frac{\mathbf{w}^{H} \mathbf{R}_{\mathrm{sp}} \mathbf{w}}{\mathbf{w}^{H} \mathbf{R}_{\mathrm{iso}} \mathbf{w}}
$$

where $T_{\mathrm{sp}}$ is the brightness temperature in the complement $\Omega_{\mathrm{sp}}$ of the solid angle subtended by the reflector. This definition is equivalent to the IEEE standard convention for spillover efficiency, which is given for a transmitter as the ratio of power intercepted by the reflector to the total radiated power, and assumes a constant spillover temperature distribution. If a more detailed spillover noise model is desired, (4) and (5) can be modified to account for a non-uniform brightness temperature distribution, such as warm ground and cooler sky above horizon. The beam radiation efficiency is

$$
\eta_{\text {rad }}=\frac{\mathbf{w}^{H} \mathbf{R}_{\text {iso }} \mathbf{w}}{\mathbf{w}^{H}\left(\mathbf{R}_{\text {iso }}+\mathbf{R}_{\text {loss }}\right) \mathbf{w}}
$$

where $\mathbf{R}_{\text {loss }}$ here assumes that the physical temperature of the

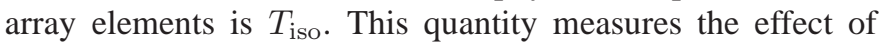
loss in the antenna elements on the system noise.

By inserting (9)-(11) into (8), the sensitivity can be expressed as

$$
\frac{A_{\mathrm{e}}}{T_{\mathrm{sys}}}=\frac{\eta_{\mathrm{rad}} \eta_{\mathrm{ap}} A_{\mathrm{ap}}}{\eta_{\mathrm{rad}}\left(1-\eta_{\mathrm{sp}}\right) T_{\mathrm{sp}}+\left(1-\eta_{\mathrm{rad}}\right) T_{\mathrm{a}}+T_{\mathrm{rec}}}
$$

where $T_{\mathrm{a}}$ is the physical temperature of the array elements and

$$
T_{\text {rec }}=T_{\text {iso }} \frac{\mathbf{w}^{H} \mathbf{R}_{\text {rec }} \mathbf{w}}{\mathbf{w}^{H}\left(\mathbf{R}_{\text {iso }}+\mathbf{R}_{\text {loss }}\right) \mathbf{w}}
$$

can be identified as a beam equivalent receiver noise temperature. To parameterize receiver noise performance as an efficiency, we can define the noise matching efficiency

$$
\eta_{\mathrm{n}}=\frac{T_{\mathrm{rec}, \mathrm{min}}}{T_{\mathrm{rec}}}
$$

where $T_{\text {rec,min }}$ is the equivalent receiver noise temperature for a single, isolated receiver chain with optimal source impedance at the input. Due to mutual coupling, $\eta_{\mathrm{n}}$ is beamformer dependent and generally less than unity.

The goal with PAF beamforming is to achieve high aperture efficiency (9) while also minimizing the spillover and receiver noise, so that the sensitivity (8) is maximized. Previous studies have suggested the use of modeled field distributions in the focal plane to infer a set of beamformer weights [16]. A more rigorous approach is the conjugate field match (CFM) beamformer $\mathbf{w}=\mathbf{a}_{\Omega}$. The CFM beamformer does not maximize sensitivity, however, and is inadequate for a high sensitivity RA instrument.

For a point signal of interest, sensitivity as defined in (8) is maximized with

$$
\mathbf{w}=\mathbf{R}_{\mathrm{n}}^{-1} \mathbf{a}_{\Omega} .
$$

Under assumptions of a single point source and no interference, this is equivalent within a scale factor to the classical max-SNR and MVDR (Capon) beamformers [14]. Typically, this type of statistically optimal beamformer is viewed as an adaptive algorithm. For RA, however, the noise environment is quasi-stationary, and $\hat{\mathbf{R}}_{\mathrm{n}}$ and $\hat{\mathbf{a}}_{\Omega_{i}}$ are available as byproducts of the calibration procedure of Section II-C. The max-sensitivity beamformer can be used in what might be termed a "fixed-adaptive" mode which is data-dependent from a calibration phase but remains constant during observations. We recommend this approach for PAF beamformer design in all fixed beamforming, even when adaptive interference canceling is not needed. By optimizing sensitivity, the best possible trade-off is achieved between performance metrics such as spillover efficiency, aperture efficiency, and receiver noise. Due to the complexities of non-identical element patterns, correlated noise, mutual coupling, and the difficulty of measuring calibration vectors on a dense grid over the full pattern, including spillover region, other traditional deterministic beamformer design techniques intended to meet a specified dish illumination pattern are simply not practical.

To illustrate these considerations, we present modeled results for a PAF on a reflector consistent with the Green Bank 20 -Meter Telescope $(f / D=0.43)$. The PAF is a 19 element hexagonal array of dipoles with 0.6 wavelength spacing at $1600 \mathrm{MHz}$ backed by a ground plane. The elements are modeled as lossless, so $\eta_{\mathrm{rad}}=1$. The field scattered by the reflector is computed using the physical optics approximation.

Results are shown in Figure 4 as a function of the minimum receiver noise temperature $T_{\text {rec,min }}$, which is a measure of the quality of the receivers. The range shown for $T_{\text {rec,min }}$ approximates a continuum of amplifier noise performance from typical communications systems to the cryo-cooled amplifiers used in radio astronomy systems. For small $T_{\text {rec,min }}$, the beamformer under-illuminates the reflector and sacrifices aperture efficiency in order to reduce spillover noise. At $T_{\text {rec }, \min }=5 \mathrm{~K}$, 

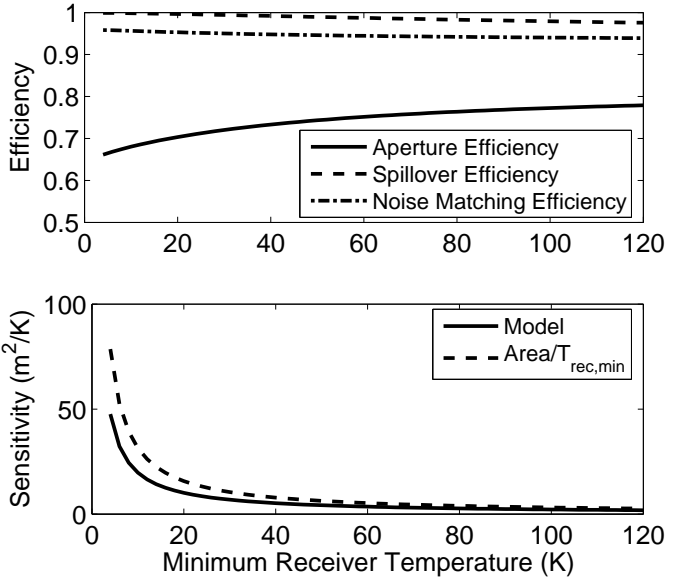

Fig. 4. Modeled beam efficiencies (top) and sensitivity (bottom) for a 19 element phased array feed and 20 meter reflector. The sensitivity bound $A_{\text {ap }} / T_{\text {rec, min }}$ (i.e., $\eta_{\text {ap }}=\eta_{\text {spill }}=\eta_{\mathrm{n}}=1$ ) is also shown

$\eta_{\mathrm{ap}}=66 \%, T_{\mathrm{sys}}=5.4 \mathrm{~K}$, and $A_{\mathrm{e}} / T_{\mathrm{sys}}=38.3 \mathrm{~m}^{2} / \mathrm{K}$ (these values are optimistic since the model neglects effects such as ohmic loss, blockage, and feed support scattering). With the suboptimal CFM beamformer, $\eta_{\mathrm{ap}}=83 \%, T_{\mathrm{sys}}=30.8 \mathrm{~K}$, and $A_{\mathrm{e}} / T_{\text {sys }}=8.5 \mathrm{~m}^{2} / \mathrm{K}$. The aperture efficiency for the optimal beamformer is lower than that of CFM, but the overall sensitivity is higher by $6.5 \mathrm{~dB}$, which highlights the need for the noise-dependent beamforming strategy described above.

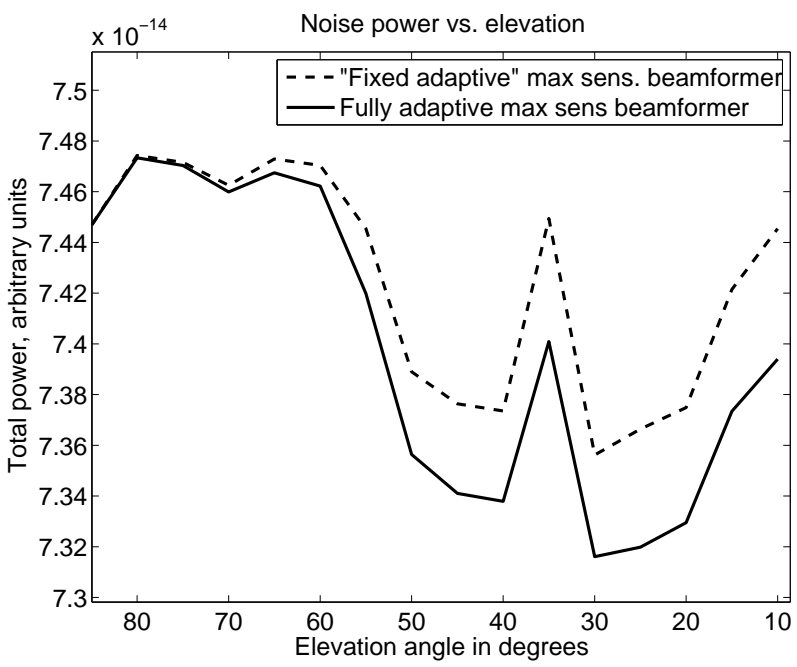

Fig. 5. PAF sensitivity optimization over pointing elevation angle from real-data experiments on the Green Bank 20-meter Telescope using the BYU/NRAO 19 element PAF. When allowed to adapt with tipping angle, the beamformer exploits anisotropy in the spillover noise field to reduce total noise power. The peak near $35^{\circ}$ is due to passing through a deep space source.

Figure 5 illustrates another promising advantage of using an adaptive PAF in RA applications. These are the first experimental results to demonstrate that a PAF can increase sensitivity by exploiting the non-istropic noise field as the dish tips to different elevation angels. With the dish pointed to zenith, the PAF spillover pattern sees warm ground. At mid elevations the upper spillover sidelobes see cold sky. At low elevations, noise in the dish main lobe begins to dominate since its oblique sight line through the atmosphere cuts through more warm air, raising the observed sky temperature. By continuously adapting to this changing noise environment with the maximum sensitivity beamformer of (16), the fully adaptive PAF is shown to have lower total noise output than when weights are fixed at the optimal solution for the zenith pointing. For elevation dependent fixed-adaptive operation, the continuously adapting beamformer weights can be precomputed from a calibration cut in elevation, and called up later from a lookup table as a function of pointing elevation. The CFM beamformer yields a minimum noise power level of 8.65 on the same scale as Figure 5 when applied to the same data set, and thus performs worse than either of the curves plotted.

\section{RFI MitigATION}

Perhaps the most compelling argument for an active, adaptive PAF as opposed to fixed beamformer weights is its potential for interference mitigation by placing spatial nulls in the direction of offending sources. As contemporary science goals increasingly require observing sources outside the traditional protected spectrum bands, a critical need is developing to deal will ubiquitous man-made interfering signals such as satellite downlink transmissions [17], [18], radar systems [19], [20], air navigation aids [21], wireless communications [22], and digital television broadcasts. A variety of mitigation methods have been studied by us and others for single dish observation, including adaptive filtering using a reference antenna [18], [23]-[25], time blanking [19], [20], and parametric signal estimation and subtraction [17]. Also, array spatial filtering has been studied by us and others for synthesis imaging with large dish arrays and beamformed aperture arrays [23], [26][30]. Post correlation interference mitigation has been used with the Parkes Telescope HIPASS horn feed array [31].

The phased array feed offers a promising new (for radio astronomy) approach to exploit the spatial structure of the interfering signal for mitigation. Any number of well known adaptive canceling beamformers or spatial filtering methods can be considered for PAF interference cancellation, including minimum variance distortionless response (MVDR), maximum SNR, minimum variance linear constraint (LCMV), subspace projection, generalized sidelobe canceler (GSC), and several robust constrained beamformers [14], [32], [33].

\section{A. Challenges for Active PAF Interference Cancellation}

Even with the potential advantages, active PAF interference canceling has to this point remained a research topic for radio astronomy. It has yet to become an operational tool at any of the world's major instruments. We identify the following key challenges that can arise specifically due to active PAF interference cancellation. These must be resolved or better understood before adaptive canceling PAFs can be embraced in the radio astronomy community.

1) Mainlobe beam pattern distortion: Adaptive beamformers must distort the desired quiescent (interference free) beam pattern in order to place deep nulls on interferers [14]. For astronomy, even modest beamshape distortions can be unacceptable. A small pointing shift in mainlobe peak response, or 


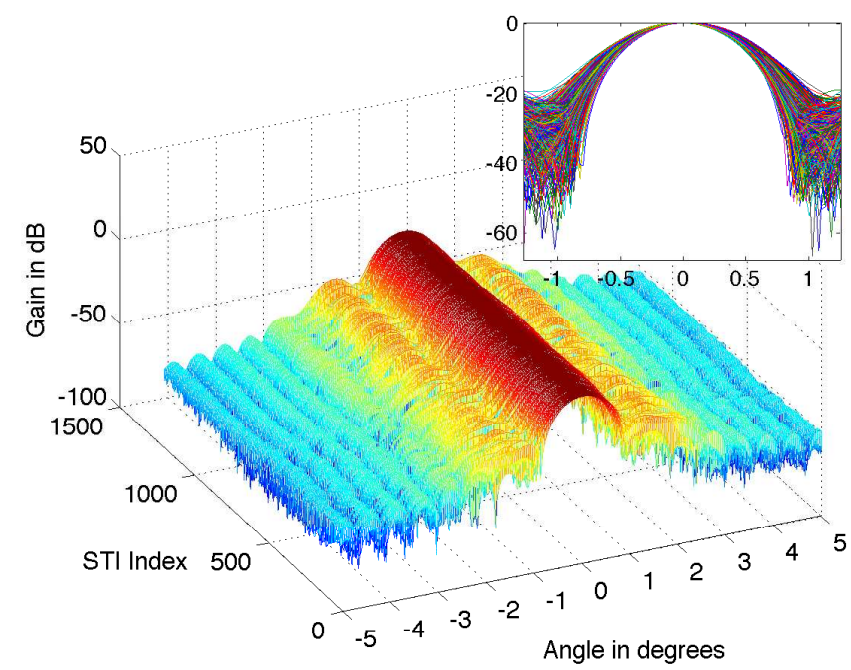

Fig. 6. LCMV beam pattern variation over time (STI index) for a 19 element PAF on a $20 \mathrm{~m}$ dish with a strong moving interferer. The pattern is for an elevation cut in the far field, combined PAF - dish response, with dish and beam pointed to zenith. Inset shows mainlobe detail with beams for all STIs plotted on the same axes. Angle is relative to boresight. The interferer followed a spiral arc in the sidelobes spanning $\left(10^{\circ}, 80^{\circ}\right)$ to $\left(70^{\circ}, 30^{\circ}\right)$ in azimuthelevation.

coma in the beam mainlobe can corrupt sensitive calibrated measurements of object brightness spatial distribution.

A potential solution is to use one of several classical constrained adaptive beamformers [14], [34], [35]. More recent developments in robust beamformers and could improve mainlobe constraint performance in the presence of calibration errors [33], [36]. These methods must be studied for suitability in the PAF, large reflector, radio astronomy application.

Figure 6 illustrates some aspects of the problem. These results are from a detailed full-wave simulation of a $20 \mathrm{~m}$ dish and 19 element PAF. INR was $40 \mathrm{~dB}$ and the interferer did not encroach on the mainlobe or near sidelobes. A single mainlobe constraint at the peak was employed. Note that there is significant variation in the mainlobe even though interference stayed in the deep sidelobes. This suggests that several additional constraints would be needed over the 2-D pattern to maintain shape.

2) Sidelobe pattern rumble: A more subtle undesirable effect is that variations in the PAF dish illumination sidelobe pattern ("pattern rumble") translate directly to an increase in the minimum detectable signal level for the radiometer. Figure 11 below illustrates the sidelobe rumble effect.

Weak astronomical sources can only be observed by integrating the received power for a long period to obtain separate low variance estimates of signal plus noise power (on source), and noise only (off source). Both signal and noise must be stable to an extreme tolerance requirement over the full integration time.

The noise field seen by the PAF is dominated by spillover region thermal ground radiation, but this is non-isotropic due to reflector blockage. Because of this anisotropy, even small variations in the PAF sidelobe structure in the spillover region can significantly perturb beamformer noise levels, causing intolerable time variation. This occurs even if the beam pattern mainlobe is held stable using constrained or robust beamformer techniques. Figure 9 below illustrates the negative effect on integrated noise floor estimates when adaptive cancelation perturbs sidelobe patterns.

No known adaptive beamformer can maintain sidelobe detailed structure while canceling a moving interferer. There are insufficient degrees of freedom to constrain the entire sidelobe pattern. A recent promising method provides both mainlobe shape and maximum sidelobe level control for in the presence of strong interference [37]. Though detailed sidelobe structure is not maintained, peak sidelobe levels are kept below a specified limit. Unfortunately the method requires calibration information for the entire constrained sidelobe region. This is impractical or impossible for the PAF fed RA telescope dish, except in simulation.

3) Cancellation null depth: The typical astronomical signal power level is $30 \mathrm{~dB}$ or more below the system noise. Canceling nulls must be deep enough to drive interference below the SOI level, i.e. below the on-source minus off-source detection limit, not just to the system noise level.

Most algorithms require a dominant interferer to form deep nulls. Minimum variance methods (MVDR, LCMV, max SNR etc.) which balance noise variance with residual interference power cannot drive a weaker interferer far below the noise floor. Zero forcing beamformers like subspace projection can drive deeper nulls, but interference subspace estimation is poor without a dominant signal, and null depth suffers. Short integration times, needed to avoid subspace smearing with moving interference, increase covariance sample estimation error which also limits null depth.

We are studying a few approaches to solve this open problem. First, we have shown that use of auxiliary antennas steered to obtain high interference-to-noise ratio (INR) data can significantly improve cancellation depth when compared to other array processing algorithms [23]. Second, low order parametric models can be used to represent moving interference covariance structure evolution over windows longer than the STI stationarity time limit which typically bounds sample covariance integration. Significant work remains to be done on this topic.

\section{B. PAF Adaptive Cancellation Methods}

We will consider LCMV beamforming and subspace projection beamforming as representative adaptive canceling algorithms. At each STI the well known LCMV weight is computed as [14]

$$
\mathbf{w}_{i, j}=\hat{\mathbf{R}}_{x, j}^{-1} \mathbf{B}_{i}\left[\mathbf{B}_{i}^{H} \hat{\mathbf{R}}_{x, j}^{-1} \mathbf{B}_{i}\right]^{-1} \mathbf{f}_{i}
$$

where columns of $\mathbf{B}_{i}$ and response vector $\mathbf{f}_{i}$ define a set of linear response constraints on the $i$-th beamformer steered to $\Omega_{i}$, such that $\mathbf{B}_{i}^{H} \mathbf{w}_{i, j}=\mathbf{f}_{i}$. These are typically used to control mainlobe shape, and can be constructed from $\hat{\mathbf{a}}_{\Omega_{i}}$ estimates provided by the calibration of Section II-C.

The subspace projection beamformer has time-varying weight vector given by

$$
\mathbf{w}_{i, j}=\mathbf{P}_{j} \overline{\mathbf{w}}_{i}, \quad \mathbf{P}_{j}=\mathbf{I}-\mathbf{U}_{d, j} \mathbf{U}_{d, j}^{H}
$$




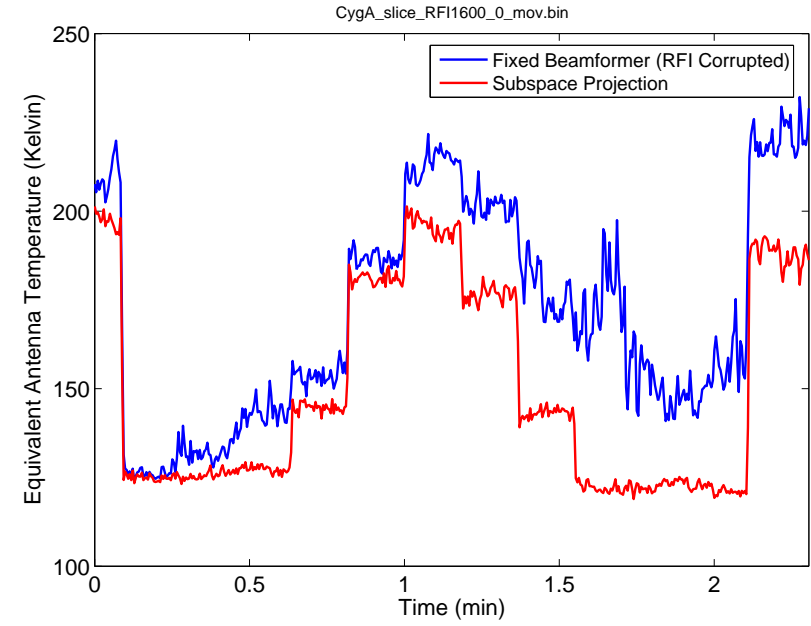

Fig. 7. Experimental results for the BYU/NRAO L-band 19 element PAF on the Green Bank 20-Meter Telescope, Nov. 2007. RFI mitigation using the subspace projection beamforming. Interference was a moving $\mathrm{CW}$ transmitter in deep reflector sidelobes, while the dish was stepped in elevation through the source, $1 / 4$ beamwidth per step. $f / D=0.43$.

where $\mathbf{P}_{j}$ is an estimate of the perpendicular projection matrix for the interference subspace, $\mathbf{V}_{j}=\left[\mathbf{v}_{1}[j L], \cdots, \mathbf{v}_{Q}[j L]\right]$. $\mathbf{U}_{d, j}$ contains normalized eigenvectors corresponding to the $Q$ largest eigenvalues in the decomposition $\hat{\mathbf{R}}_{x, j}=\mathbf{U}_{j} \boldsymbol{\Lambda} \mathbf{U}_{j}^{H}$ such that $\mathbf{U}_{j}=\left[\mathbf{U}_{d, j} \mid \mathbf{U}_{s+\mathbf{n}, j}\right]$.

Fixed beamforming weight $\overline{\mathbf{w}}_{i}$ is designed for the desired quiescent beam response with mainlobe steered to $\Omega_{i}$. Due to the difficulty in calibrating the PAF response over the entire spillover region, we advocate using the "fixed adaptive" beamformer of (16) using $\hat{\mathbf{R}}_{n}$ and $\hat{\mathbf{a}}_{\Omega_{i}}$ taken from the calibration procedure of Section II-C to calculate $\overline{\mathbf{w}}_{i}$. Alternatively, to provide additional mainlobe shape constraints in $\overline{\mathbf{w}}_{i},(17)$ can be used, replacing $\hat{\mathbf{R}}_{x, j}$ with off-source calibration $\hat{\mathbf{R}}_{n}$.

Figure 7 presents new observations obtained during our recent PAF experiments on the NRAO Green Bank 20-Meter Telescope. The improvement in signal of interest response using subspace projection beamforming to reject an interferer is quite significant.

\section{Correcting Pattern Rumble Bias}

We have recently introduced an algorithm [38], [39] which adapts the corrected spatial filtering approach of Leshem and van der Veen [40] to the problem of power spectral density (PSD) estimation with a sensor array. When conventional PSD algorithms are applied to the output of an adaptive beamformer, as in Figure 2, a significant bias error appears in the spectrum due to beampattern variation (pattern rumble) while tracking a moving interference. The new bias corrected subspace projection beamformer removes this error.

Instead of the cascaded beamformer and PSD estimator as seen in Figure 2, we proposed a joint adaptive canceler and

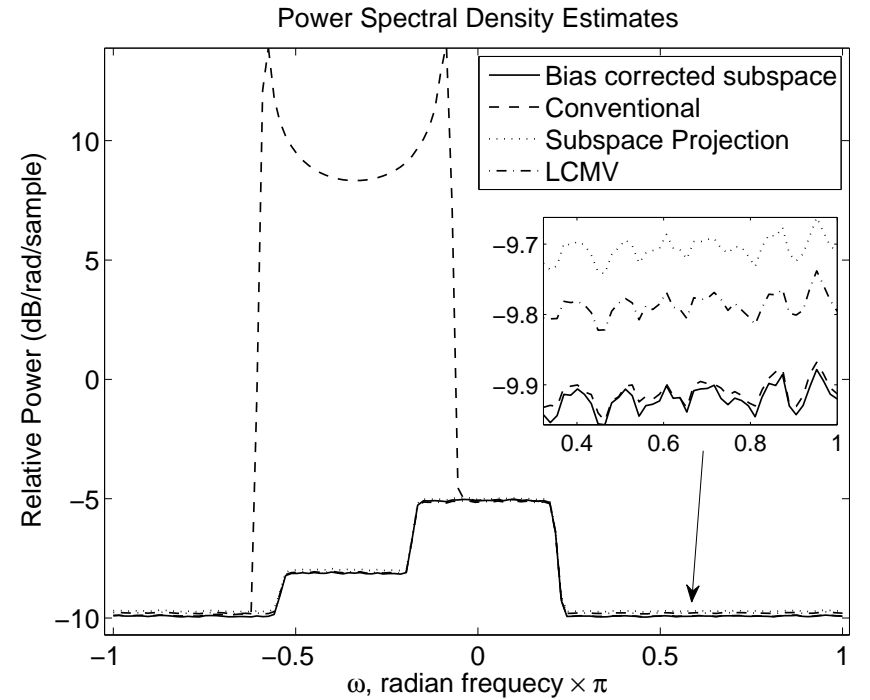

Fig. 8. Simulated pattern rumble bias correction results at $1600 \mathrm{MHz}$ for a 19 element PAF on a $20 \mathrm{~m}$ diameter dish with $f / D=0.33$. The interferer is the dominant FM modulated tone seen centered at $\omega=-0.37 \pi$ in the conventional fixed beamformer PSD response. Inset shows expanded noise floor detail. PSD levels are normalized for equal response to the SOI. The dish and beam are pointed to zenith. The interferer followed a spiral arc in the sidelobes spanning $\left(10^{\circ}, 80^{\circ}\right)$ to $\left(70^{\circ}, 30^{\circ}\right)$ in azimuth-elevation during 4700 STI periods of $L=1024$ samples each.

estimator as follows

$$
\begin{aligned}
\hat{\mathbf{S}}_{y_{i}}^{T}= & \frac{\alpha}{M}\left(\overline{\mathbf{w}}_{i}^{H} \otimes \overline{\mathbf{w}}_{i}^{T}\right) \mathbf{C}^{-1} \sum_{j=0}^{M-1}\left[\left(\mathbf{P}_{j}\left(\mathrm{FFT}_{N}\left\{\mathbf{X}_{j} \odot \boldsymbol{\Gamma}\right\}\right)\right)\right. \\
& \left.\circ\left(\mathbf{P}_{j}\left(\mathrm{FFT}_{N}\left\{\mathbf{X}_{j} \odot \boldsymbol{\Gamma}\right\}\right)\right)^{*}\right] \\
\mathbf{C}= & \frac{1}{M} \sum_{j=0}^{M-1}\left(\mathbf{P}_{j} \otimes \mathbf{P}_{j}^{*}\right)
\end{aligned}
$$

where $\mathrm{FFT}_{N}\{\cdot\}$ denotes the $N$ point one-dimensional fast Fourier transform along matrix rows, $M$ is the number of FFT windows which are averaged, and $\overline{\mathbf{w}}_{i}$ and $\mathbf{P}_{j}$ are defined in (18). Each row of $\boldsymbol{\Gamma}$ is a copy of the tapered window (e.g. Hamming) used to reduce spectral leakage, and $\alpha$ is a scale factor to correct bulk scale bias introduced by $\Gamma$.

It was shown that for a small uniform line array (19) produces an "effective beam pattern," on average over the full integration interval, which exactly matches the quiescent beamformer that non-adaptive $\overline{\mathbf{w}}_{i}$ alone would produce [39]. Pattern-rumble-induced PSD bias is also removed. These corrections are accomplished while canceling the interferer. No other known adaptive array processor is capable of this.

Figures 8-11 present new results of a detailed numerical simulation for the electromagnetic response of a 19 element PAF on a $20 \mathrm{~m}$ dish with a strong moving interferer seen deep in the dish sidelobes. The algorithm succeeds in maintaining a perfect effective beamshape for both the far dish pattern on the sky, and the PAF illumination pattern on the dish. An $f / D=0.33$ was chosen for the simulation since it results in somewhat more pattern rumble than the $f / D=0.43$ of the Green Bank 20-Meter Telescope.

These results are the first to demonstrate that bias correction is effective in a PAF and large reflector environment, including 


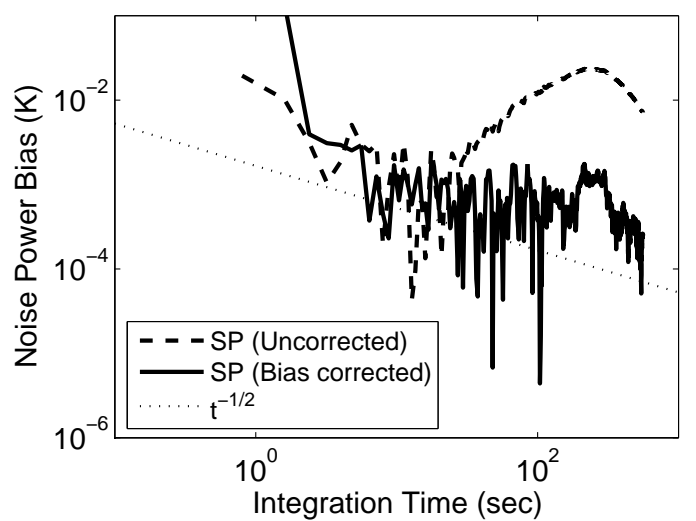

Fig. 9. Difference (error) between cumulative integrated beamformer noise power estimate and true noise power for a 19 element PAF model with 20 meter reflector, $f / D=0.33$. With bias correction, increased integration time yields a noise power estimate which converges at the theoretical rate to the expected noise power (the $t^{-1 / 2}$ sample error stdv. curve). Without bias correction additional integration time fails to reduce error variance.

non-identical, complex element response patterns, reflector beam focusing effects, strong mutual coupling, and correlated receiver and spillover noise. Previously, bias correction was only known to fully correct the effective beampattern for a uniform line array. The electromagnetic simulation used finite element modeling for the PAF, microwave network theory for receiver noise, and physical optics for the reflector.

Pattern rumble in the spillover region has biased the noise floor PSD estimate high, even with long integration, for both subspace projection and LCMV cancelers. The bias corrected algorithm solves the problem, bringing the noise floor down to the level that would be seen by the conventional fixed weight beamformer in the absence of interference.

For this experiment the total in-band power INR was +40 $\mathrm{dB}$. The SOI is a multiband filtered Gaussian random process, seen as a step pattern between $-0.5 \pi \leq \omega \leq 0.25 \pi$. SNR was $-5 \mathrm{~dB}$, which though higher than a typical deep space source, provides a clearly readable illustration. The conventional beamformer PSD is completely dominated by the interferer, and would fail at estimating SOI spectrum inside the interference band. LCMV and subspace projection both effectively cancel interference, but raise the noise floor as seen in the inset. The bias corrected PSD completely cancels interference but has the same noise floor level as the nonadaptive conventional beamformer, outside of the interference spectrum.

The demonstrated level of noise floor bias due to uncorrected interference cancelation seems small, but would be catastrophic when trying to detect $\mathrm{mJy}$ and $\mu \mathrm{Jy}$ level space sources. This is particularly true since with a moving and perhaps intermittent interferer, the bias levels in on-source and off-source PSDs would be different.

Figure 9 shows the importance of bias correction for long integration detection. The simulation scenario matches Figure 8 , but with no SOI. Total noise power sample estimates in beamformer output, $y_{i}[n]$, are shown for subspace projection with a strong interferer present. With increasing integration time the uncorrected subspace projection fails to converge due to pattern rumble bias. On the other hand, bias corrected

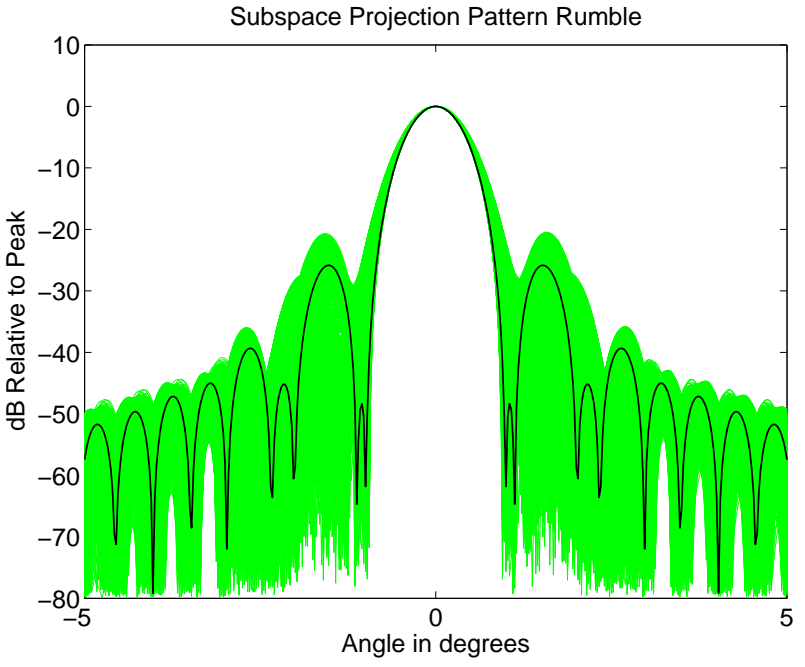

Fig. 10. Main beam (dish and PAF) pattern rumble with subspace projection algorithm over many STI windows for 19 element PAF model with 20 meter reflector, $f / D=0.33$. The bias corrected effective response pattern is shown with the solid black line. It exactly matches the quiescent, fixed weight conventional beamformer.

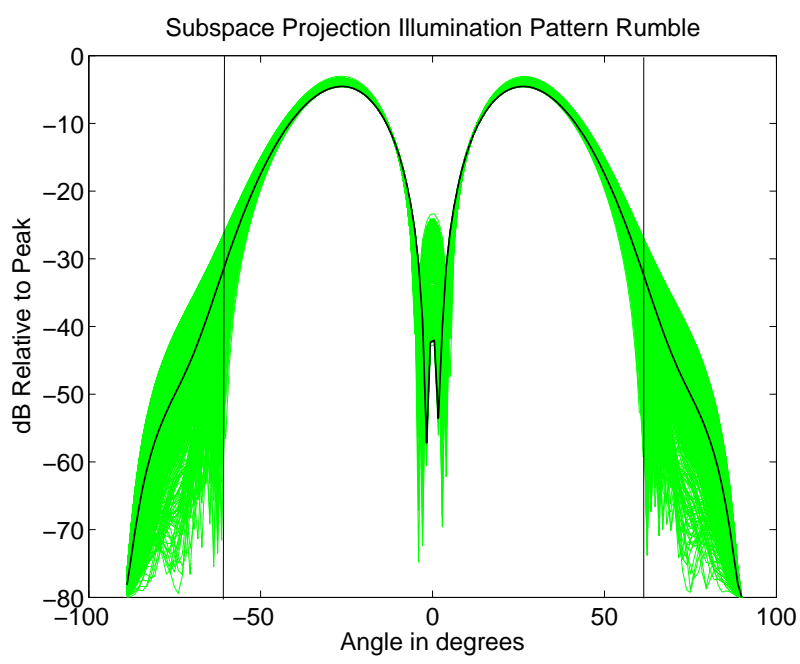

Fig. 11. Illumination pattern on the dish for the bare PAF array with subspace projection algorithm over many STI windows for 19 element PAF model with 20 meter reflector, $f / D=0.33$. Vertical lines denote the dish rim. The bias corrected effective response pattern is shown with the solid black line. It exactly matches the quiescent, fixed weight conventional beamformer.

subspace projection converges at the theoretical $t^{-1 / 2}$ rate toward the true power. For the example source described in Section III, the signal of interest would not be detectable until the integrated noise power estimate bias is below $3.4 \mathrm{mK}$.

Figures 10 and 11 show another significant aspect of bias correction. Simulated elevation cuts through the main beam (combined dish and PAF) and dish illumination pattern of the bare PAF array are shown. The same moving interferer scenario as in Figure 8 is used with the 19 element PAF and $20 \mathrm{~m}$ dish. Patterns for each STI are over-plotted on the same axes to illustrate the range of variation over time. The solid black curve represents the effective bias corrected beampattern over the full integration window. It exactly matches the quiescent beampattern formed using fixed weights, $\overline{\mathbf{w}}_{i}$.

The effective bias corrected pattern is computed by storing 
projection matrices, $\mathbf{P}_{j}$, formed during a run with the signal plus noise plus interference data. A new probing data set, $\mathbf{X}_{\Omega_{k}, j}, 0 \leq j<M$, is generated with a single far-field point source at angle $\Omega_{k}$, and no noise or interference. Effective response in direction $\Omega_{i}$ is computed using the previously saved $\mathbf{P}_{j}$ with probe signal $\mathbf{X}_{\Omega_{k}, j}$ in (19). The process is repeated for each plotted arrival angle.

The wide variation in main beam (dish and PAF) pattern over the STIs is entirely unacceptable for the precise radiometric observations needed for credible astronomical science. This is why astronomers have not embraced adaptive array processing to deal with their interference mitigation problems. The bias corrected effective beam response resolves these concerns for PSD observations.

An interesting aspect of Figure 11 is the "hole" in the center of the pattern. The quiescent beamformer fixed weights were computed using (16) with calibration data. Apparently it is more important in the optimization to form sharper transitions to low sidelobe response at the dish edge than to enforce a smooth pattern across the dish. The hole covers only a small central area of the dish under the obscuring feed array. The sensitivity achieved with this solution is higher than any other illumination pattern possible for the 19 element PAF, including smooth response, hole-free patterns. For larger arrays, a characteristic FIR filter passband ripple pattern appears.

\section{Vi. CONClusions}

Signal processing for radio telescopic phased array feeds offers a rich and challenging regime for algorithm development. Due to the extremely tight system tolerances and low noise requirements for astronomical observations, an accurate signal and noise model is required in order to assess the key factors that affect beamformer performance. This is especially true in regard to correlated spillover and receiver noise. In order to characterize the performance of a given beamformer, we have extended the standard definitions of antenna figures of merit, such as aperture efficiency, to an array feed. Even for non-adaptive beamforming, statistically optimal beamformer solutions will likely be required each time the array is recalibrated.

Of particular importance is the gain and pattern stability required for radiometric detection of weak signals. In the presence of interferers, adaptive algorithms which have adequate performance for communications applications are unusable for radio astronomy. The well known adaptive cancelers can lead to a system power bias which is small in absolute terms, but for astronomical observations would completely obscure weak signals of interest in many cases. We presented modeled results for a bias correction algorithm which removes the bias caused by pattern rumble associated with adaptive cancellation. Our initial experimental results are promising, but resolving the remaining open technical questions will require cooperative research in several disciplines, including sensor array processing, real-time signal processing systems, electromagnetics, antenna design, and astronomy.

\section{APPENDIX}

The following notation, operators and identities are used:
1) $\mathrm{E}\{\mathbf{A}\}$ : Expected value of random $\mathbf{A}$.

2) $\bar{E}_{m}(\Omega)$ : Far-field electric field pattern at spherical angle $\Omega$ due to array element $\mathrm{m}$.

3) $\lfloor a\rfloor$ : Floor operation, rounding toward zero.

4) $a^{*}$ : complex conjugate of $a$.

5) $\mathbf{A}^{T}, \mathbf{A}^{H}$ : transpose and conjugate transpose of $\mathbf{A}$.

6) $\hat{\mathbf{A}}$ : estimate of $\mathbf{A}$.

7) $\mathbf{A} \otimes \mathbf{B}:$ Kronecker matrix product.

8) $\mathbf{A} \circ \mathbf{B}=\left[\mathbf{a}_{1} \otimes \mathbf{b}_{1}, \cdots, \mathbf{a}_{N} \otimes \mathbf{b}_{N}\right]$, Khatri-Rao product.

9) $\mathbf{A} \odot \mathbf{B}$ : element-wise, or Hadamard array product.

\section{REFERENCES}

[1] John D. Kraus, Radio Astronomy, Second Ed., Cygnus-Quasar Books, Powell, Ohio, 1986.

[2] S. Snezana and NAIC-NRAO Summer School on Single Dish Radio Astronomy, Single-dish radio astronomy : techniques and applications : proceedings of the NAIC-NRAO Summer School, Astronomical Society of the Pacific, San Francisco, 2002.

[3] A.R. Thompson, J.M. Moran, and G.W. Swenson Jr., Interferometry and Synthesis in Radio Astronomy, Second Edition, Wiley-Interscience, New York, 2001.

[4] L. Staveley-Smith et al, "The Parkes 21-cm multibeam receiver," Publications of the Astronomical Society of Australia, PASA, vol. 13, pp. $243-, 1996$, see also http://www.atnf.csiro.au/research/multibeam/.

[5] J.R. Fisher and R.F. Bradley, "Full sampling array feeds for radio telescopes," Proceedings of the SPIE, Radio Telescopes, vol. 4015, pp. 308-318, 2000.

[6] Chad K. Hansen, Karl F. Warnick, Brian D. Jeffs, J. Richard Fisher, and Richard Bradley, "Interference mitigation using a focal plane array," Radio Science, vol. 40, doi:10.1029/2004RS003138, no. 5, June 2005.

[7] Bruce Veidt and Peter Dewdney, "A phased-array feed demonstrator for radio telescopes," in Proc. URSI General Assembly, 2005.

[8] R. Maaskant, M. V. Ivashina, R. Mittra, and N. T. Huang, "Parallel FDTD modeling of a focal plane array with Vivaldi elements on the highly parallel LOFAR BlueGene/L supercomputer," in Proc. IEEE Antennas and Propagation Symposium, Albuquerque, NM, June 20-25 2006, pp. $3861-3864$.

[9] International SKA Steering Committee (ISSC), SKA project home page, 2008, http://www.skatelescope.org/.

[10] J.R. Nagel, K.F. Warnick, B.D. Jeffs, J.R. Fisher, and R. Bradley, "Experimental verification of radio frequency interference mitigation with a focal plane array feed," Radio Science, vol. 42, 2007, RS6013, doi:10.1029/2007RS003630.

[11] K. F. Warnick and B. D. Jeffs, "Beam efficiencies and system temperature for a focal plane array," Tech. Rep. http://hdl.handle.net/1877/588, Brigham Young University, Provo, UT, Nov. 2007.

[12] K. F. Warnick and M. A. Jensen, "Optimal noise matching for mutuallycoupled arrays," IEEE Transactions on Antennas and Propagation, vol. 55, no. 6, pp. 1726-1731, June 2007.

[13] K. F. Warnick and M. A. Jensen, "Effect of mutual coupling on interference mitigation with a focal plane array," IEEE Trans. Ant. Propag., vol. 53, no. 8, pp. 2490-2498, Aug. 2005.

[14] H.L. Van Trees, Detection, Estimation, and Modulation Theory, Part IV, Optimum Array Processing, John Wiley and Sons, 2002.

[15] K. F. Warnick and B. D. Jeffs, "Gain and aperture efficiency for a reflector antenna with an array feed," IEEE Antennas and Wireless Propagation Letters, vol. 5, no. 1, pp. 499-502, 2006.

[16] M. Ivashina and C.G.M van't Klooster, "Focal fields in reflector antennas and associated array feed synthesis for high efficiency multibeam performances," in Proceedings of the ESTEC Antenna Workshop, Noordwijk, The Netherlands, Sept. 2002.

[17] J.F. Bell S.W. Ellingson, J. Bunton, "Removal of the GLONASS C/A signal from $\mathrm{OH}$ spectral line observations using a parametric modeling technique," Astrophysical J. Supplement, vol. 135, pp. 87-93, July 2001.

[18] A.J. Poulsen, B.D. Jeffs, K.F. Warnick, and J.R. Fisher, "Programmable real-time cancellation of GLONASS interference with the Green Bank telescope," Astronomical Journal, vol. 130, no. 6, pp. 2916 - 2927, Dec. 2005.

[19] B.D. Jeffs, W. Lazarte, and J.R. Fisher, "Bayesian detection of radar interference in radio astronomy," Radio Science, vol. 41, pp. doi:10.1029/2005RS003400, June 2006. 
[20] S.W. Ellingson and G.A. Hampson, "Mitigation of radar interfernece in L-band radio astronomy," The Astrophysical Journal Suplement Series, vol. 147, pp. 167-176, 2003.

[21] Q. Zhang, Y. Zheng, S.G. Wilson, J.R. Fisher, and R. Bradley, "Excision of distance measuring equipment interference from radio astronomy signals," The Astronomical Journal, vol. 129, no. 6, pp. 2933-2939, June 2005.

[22] A.J. Boonstra, A. Leshem, A-J van der Veen, A. Kokkeler, and G. Schoonderbeek, "The effect of blanking of TDMA interference on radio-astronomical observations: experimental results," in Proc. of the IEEE International Conf. on Acoust., Speech, and Signal Processing, 2000, vol. 6, pp. 3546-3549.

[23] B.D. Jeffs, L. Li, and K.F. Wanick, "Auxiliary antenna assisted interference mitigation for radio astronomy arrays," IEEE Trans. Signal Processing, vol. 53, no. 2, pp. 439-451, Feb. 2005.

[24] C. Barnbaum and R.F. Bradley, "A new approach to interference excision in radio astronomy: Real-time adaptive cancellation," Astronomical Journal, vol. 116, pp. 2598-2614, Nov. 1998.

[25] F.H. Briggs, J.F. Bell, and J. Kesteven, "Removing radio interference from contaminated astronomical spectra using an independent reference signal and closure relations," Astronomical Journal, vol. 120, pp. 33513361, Aug. 2000.

[26] S. van der Tol and A.-J. van der Veen, "Performance analysis of spatial filtering of rf interference in radio astronomy," IEEE Trans. Signal Processing, vol. 53, no. 3, Mar. 2005.

[27] A. Leshem and A.-J. van der Veen, "Radio-astronomical imaging in the presence of strong radio interference," IEEE Trans. Inform. Theory, vol. 46, no. 5, pp. 1730-1747, Aug. 2000.

[28] A.J. Boonstra, S.J. Wijnholds, S. v.d. Tol, and B. Jeffs, "Calibration, sensitivity, and rfi mitigation requirements for lofar," in Proc. of the IEEE International Conf. on Acoust., Speech, and Signal Processing, Mar. 2005, vol. V, pp. 869-872.

[29] S.W. Ellingson, "Beamforming and interference canceling with very large wideband arrays," IEEE Trans. Antennas Propagat., vol. 51, no. 6, pp. 1338-1346, June 2003.

[30] G.C. Bower, "Radio frequency interference mitigation for detection of extended sources with an interferometer," Radio Science, vol. 40, no. RS5S07, Dec. 2005, doi:10.1029/2004RS003141.

[31] D.G. Barnes, F.H. Briggs, and M.R. Calabretta, "Postcorrelation ripple removal and radio frequency interference rejection for parkes telescope survey data," Radio Science, vol. 40, no. RS5S13, Dec. 2005, doi:10.1029/2004RS003139.

[32] S.W. Ellingson and G.A. Hampson, "A subspace-tracking approach to interference nulling for phased array-based radio telescopes," IEEE Trans. Antennas Propagat., vol. 50, no. 1, pp. 25-30, Jan. 2002.

[33] Jian Li and Petre Stoica, Eds., Robust Adaptive Beamforming, WileyIntersicience, Hoboken, New Jersey, 2005.

[34] S.P. Applebaum and D.J. Chapman, "Adaptive arrays with main beam constraints," IEEE Trans. Antennas Propagat., vol. AP-24, pp. 650-662, Sept. 1976.

[35] C-Y. Tseng and L.J. Griffiths, "A unified approach to the design of linear constraints in minimum variance adaptive beamformers," IEEE Trans. on Antennas and Propagation, vol. AP-40, pp. 1533 -1542, Dec. 1992.

[36] A.B. Gershman, U. Nickel, and J.F. Böhme, "Adaptive beamforming algorithms with robustness against jammer motion," IEEE Trans. on Signal Proc., vol. 45, no. 3, pp. 1878-1885, July 1997.

[37] J. Li, Y. Xie, P. Stoica, X. Zheng, and J. Ward, "Beampattern synthesis via a matrix approach for signal power estimation," IEEE Trans. Signal Processing, vol. 55, no. 12, pp. 5643-5657, Dec. 2007.

[38] B.D. Jeffs and K.F. Warnick, "Bias corrected psd estimation with an interference canceling array," in Proc. of the IEEE International Conf. on Acoust., Speech, and Signal Processing, Honolulu, Apr. 2007, vol. II, pp. 1145-1148.

[39] B.D. Jeffs and K.F. Warnick, "Bias corrected psd estimation for an adaptive array with moving interference," IEEE Trans. Signal Processing, vol. 56, pp. doi:10.1109/TSP.2008.919637, 2008.

[40] A. Leshem, A.-J. van der Veen, and A.-J. Boonstra, "Multichannel interference mitigation techniques in radio astronomy," Astrophysical Journal Supplements, vol. 131, no. 1, pp. 355-374, 2000.

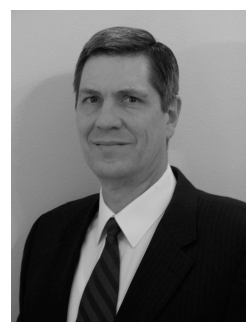

Brian D. Jeffs (M'90-SM'02) received B.S. (magna cum laude) and M.S. degrees in electrical engineering from Brigham Young University in 1978 and 1982 respectively. He received the Ph.D. degree from the University of Southern California in 1989. He is currently an associate professor in the Department of Electrical and Computer Engineering at Brigham Young University, where he lectures in the areas of digital signal processing, digital image processing, and probability theory. Current research interests include array signal processing for radio astronomy, RF interference mitigation, MIMO wireless communications, and digital image restoration. Previous employment includes Hughes Aircraft Company where he served as a sonar signal processing systems engineer in the antisubmarine warfare group. Dr. Jeffs was a Vice General Chair for IEEE ICASSP-2001, held in Salt Lake City Utah. He was a member of the executive organizing committee for the 1998 IEEE DSP Workshop, and served several years as chair of the Utah Chapter of the IEEE Communications and Signal Processing Societies.

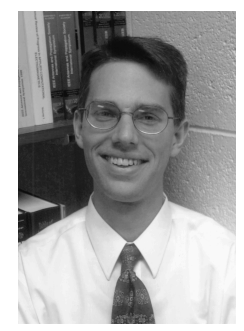

Karl F. Warnick (S'95-M'98-SM'04) received the B.S. degree (magna cum laude) with University Honors and the Ph.D. degree from Brigham Young University in 1994 and 1997, respectively. From 1998 to 2000, he was a Postdoctoral Research Associate in the Center for Computational Electromagnetics at the University of Illinois at Urbana-Champaign. Since 2000, he has been a faculty member in the Department of Electrical and Computer Engineering at Brigham Young, where he is currently an Associate Professor. He has authored a book, Problem Solving in Electromagnetics, Microwave Circuits, and Antenna Design for Communications Engineering (Artech House). Research interests include array antenna systems, computational electromagnetics, remote sensing, and inverse scattering. Dr. Warnick was a recipient of the NSF Graduate Research Fellowship, Outstanding Faculty Member award for Electrical and Computer Engineering in 2005, and the BYU Young Scholar Award (2007), and served as Technical Program Co-Chair for the 2007 IEEE International Symposium on Antennas and Propagation.

Jonthan Landon, Jacob Waldron, and David Jones each received the B.S. degree from Brigham Young University in 2006. Mr. Landon is currently pursuing the Ph.D. degree, while Mr. Waldron and Mr. Jones will complete their M.S. degrees in 2008, all in the Department of Electrical and Computer Engineering at BYU. Mr. Landon has interests in array signal processing theory and systems, and was lead graduate engineer of platform development for the Green Bank 20-Meter Telescope experiment reported in this paper. Mr. Waldron's interests include microwave antennas and circuits, and he constructed the 19 element experimental array. Mr. Jones' interests include computational electromagnetics, and he developed numerical simulation code for array and dish modeling.

J. Richard Fisher (M'74) received the B.S. degree in physics from Pennsylvania State University in 1965, and the Ph.D. degree in astronomy from University of Maryland in 1972 . He has been with the National Radio Astronomy Observatory since 1972, where he current serves as Technical Research and Development Leader at the NRAO Charlottesville, VA campus. He has been a visiting researcher at radio astronomy laboratories in Australia and India. His research interests include RFI mitigation, single dish phased array feeds, and instrument development for radio astronomy.

Roger D. Norrod (M) received the B.S. and M.S. degrees in Electrical Engineering from Tennessee Technological University in 1973 and 1979 respectively. Since 1979 he has been an electronics engineer at the National Radio Astronomy Observatory, Green Bank WV. His activities there have included cryogenic LNA design, VLBA receiver design, head of 100-Meter Green Bank Telescope Antenna Optics group, and manager of NRAO systems development for the GBT. He has previously worked at Texas Instruments, Inc., and Scientific Communications, Inc. as a radar applications and RF instrumentation engineer. 